\title{
Flexible Needle Steering in Moving Biological Tissue With Motion Compensation Using Ultrasound and Force Feedback
}

\author{
Jason Chevrie $^{(\mathbb{0}}$, Navid Shahriari ${ }^{(\mathbb{1})}$, Marie Babel, Alexandre Krupa $\left.{ }^{(}\right)$, and Sarthak Misra
}

\begin{abstract}
Needle insertion procedures under ultrasound guidance are commonly used for diagnosis and therapy. It is often critical to accurately reach a targeted region, and this can be difficult to achieve due to intraoperative tissue motion. In this letter, we present a method to steer a beveled-tip flexible needle toward a target embedded in moving tissue. Needle steering is performed using a needle insertion device attached to a robot arm. Closedloop 3-D steering of the needle is achieved using tracking of an artificial target in 2-D ultrasound images and tracking of the needle tip position and orientation with an electromagnetic tracker. Tissue motion compensation is performed using force feedback to reduce targeting error and forces applied to the tissue. The method uses a mechanics-based interaction model that is updated online. A novel control law using task functions is proposed to fuse motion compensation, steering via base manipulation and tip-based steering. Validation of the tracking and steering algorithms are performed in gelatin phantom and bovine liver. Tissue motion up to $15 \mathrm{~mm}$ is applied and average targeting error is $1.2 \pm 0.8 \mathrm{~mm}$ and $2.5 \pm 0.7 \mathrm{~mm}$ in gelatin and liver, respectively, which is sufficiently accurate for commonly performed needle insertion procedures.
\end{abstract}

Manuscript received September 10, 2017; accepted February 9, 2018. Date of publication February 27, 2018; date of current version March 28, 2018. This letter was recommended for publication by Associate Editor H. Liao and Editor K. Masamune upon evaluation of the reviewers' comments. This work was supported by funds from the Samenwerkingsverband Noord-Nederland (SNN) Program (Project RICIBION-Robotic Interventions using CT-Images for Biopsies of Lung Nodules), University Medical Center Groningen (UMCG), European Research Council (ERC) under the European Union's Horizon 2020 Research and Innovation programme (Grant 638428 - project ROBOTAR: RobotAssisted Flexible Needle Steering for Targeted Delivery of Magnetic Agents), Rennes Métropole and Réseau Franco-Néerlandais (RFN). (Jason Chevrie and Navid Shahriari contributed equally to this work.) (Corresponding author: Jason Chevrie.)

J. Chevrie and A. Krupa are with Univ Rennes, Inria, CNRS and IRISA, F-35000 Rennes, France (e-mail: jason.chevrie@irisa.fr; alexandre. krupa@inria.fr).

N. Shahriari is with the Surgical Robotics Laboratory, Department of Biomechanical Engineering, University of Twente, 7522 LW Enschede, The Netherlands, and also with the Center for Medical Imaging-North East Netherlands, University Medical Center Groningen, University of Groningen, 9713 AV Groningen, The Netherlands (e-mail: n.shahriari@utwente.nl).

M. Babel is with Univ Rennes, INSA, CNRS, Inria and IRISA, F-35000 Rennes, France (e-mail: marie.babel@irisa.fr).

S. Misra is with the Surgical Robotics Laboratory, Department of Biomechanical Engineering, University of Twente, 7522 LW Enschede, The Netherlands, and also with the Center for Medical Imaging-North East Netherlands and the Department of Biomedical Engineering, University Medical Centre Groningen, University of Groningen, 9713 AV Groningen, The Netherlands (e-mail: s.misra@utwente.nl).

This letter has supplemental downloadable multimedia material available at http://ieeexplore.ieee.org, provided by the authors. The Supplementary Materials contain a video showing an example of results obtained during the validation of our control method. This material is $9.52 \mathrm{MB}$ in size.

Digital Object Identifier 10.1109/LRA.2018.2809484
Index Terms-Surgical robotics, steerable catheters/needles, computer vision for medical robotics, medical robots and systems.

\section{INTRODUCTION}

$\mathbf{P}$ ERCUTANEOUS needle insertion procedures are commonly used for diagnosis (e.g. biopsy) and therapy (e.g. brachytherapy). Success of the procedures highly depends on accurate placement of the needle, which is challenging in presence of physiological motion. Patient breathing induces motion to the tissues near the diaphragm, such as liver or lungs. Therefore, breathing instructions are given to the patients and they are usually asked to hold their breath during the insertion [1]. However, this is not always possible for patients that may have poor breathing function. This can lead to mis-targeting, which increases the number of required needle insertions and the risks of complications. Needle insertion are usually performed under the guidance of different imaging modalities such as ultrasound (US), computed tomography (CT) or magnetic resonance imaging (MRI). CT and MRI offer high-contrast images, however, their acquisition time is not suitable for real-time applications. On the other hand, US imaging provides higher frame rate at the cost of low-contrast and noisy images. In order to perform percutaneous needle insertions accurately, researchers suggest to use robotic systems, which will be discussed in the following sections.

\section{A. Related Work}

1) Needle Steering: There are two main methods discussed in literature to steer a needle in soft tissue. The first method, known as base manipulation, applies lateral translations and rotations to the base of the needle. This creates needle deflection and causes the needle to push laterally on the tissue, and as a result the trajectory of the needle tip can be controlled. It requires a model of the interaction between the needle and the tissue to compute the amount of bending necessary to obtain the desired tip trajectory. The needle-tissue interaction can be modeled using finite element modeling or beam theory with local virtual springs [2], [3]. In order to avoid the large forces applied on the tissue, especially for deep insertions, tip steering method is used. In this method, the lateral forces created at the tip of the needle are used to steer the needle. These forces are typically obtained by an asymmetric design of the needle 
tip, such as a beveled or pre-bent tip. In this case, targeting can be achieved by orienting the force such that the needle deflects towards the target. This is often done with bevel-tipped needles by rotating the needle around its shaft such that the bevel points towards the target [4]. The radius of curvature is, however, more difficult to control, since it depends on the geometry of the tip and the relative stiffness between the needle and the tissue. The effective value of the trajectory curvature can be reduced for bevel-tipped needles using the duty cycling control method [5], [6]. Special tips have also been designed, such as an actuated tip that allows a control of both force orientation and magnitude without rotation of the needle shaft [7]. Lateral base manipulation and duty cycling control were also used together alternatively depending on the alignment of the needle with the target [8].

2) Motion Compensation: Physiological motion of the patient can induce tissue motion during the needle insertion procedure. In addition to target motion, damage to the tissue can arise if the tissue motion is large while the needle is maintained fix by a robotic needle holder. Therefore, motion compensation is necessary to limit the risk of tearing the tissue. Predictive control can be used to compensate for periodic motions, such as breathing or heart beating. This requires the position of the tissue as a feedback to be able to predict its future motion. For instance, cameras and visual markers can be used to track the surface of the body [9]. Yuen et al. used 3D US to performed 1D motion compensation for beating heart surgery [10]. Harmonic motion estimation was first used to estimate the motion of the mitral annulus before the tool begins interacting with it. After contact has been made, force control was performed by the use of a force sensor located at the tip of the tool. Impedance or admittance control are often used to perform such compensation since tissue damage can directly be avoided by reducing the force applied to the tissue. Atashzar et al. attached a force sensor to a needle holder which is maintained in contact with the surface of the tissue during the insertion, allowing to follow the motion of the tissue [11]. While axial tissue motion could be accurately compensated, lateral tissue cutting may still occur in such configuration if the tissue slips laterally with respect to the sensor. Kim et al. also compensated lateral tissue motion using a force sensor attached between the manipulator and the needle [12]. However tissue motion compensation was performed alone, without needle insertion towards a target. The research methods mentioned above for motion tracking and compensation are not easy to integrate in a clinical environment. These methods use visual markers with external tracking systems or they have direct sensor contact with the patient at the incision point. Herein lies the motivation to develop a motion compensation algorithm to be applicable for clinical environment.

3) Real-Time Ultrasound Target Tracking: Physiological motions within the body induce motion of the targeted lesion. In order to steer the needle to the target accurately, the location of the target should be tracked during the procedure. There has been a lot of developments on US needle tracking in needle steering domain. Researchers used 2D [13]-[15] and 3D [16][19] US to track the needle. There has also been several studies where US images were used for target tracking purposes.

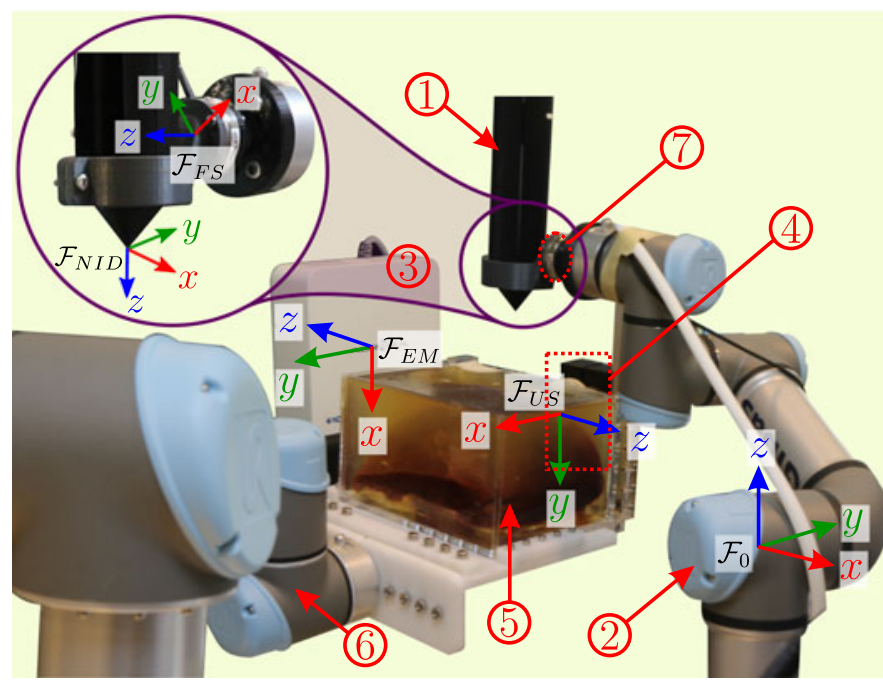

Fig. 1. A beveled-tip needle is inserted in soft tissue using a needle insertion device (1) with the reference frame $\left\{\mathcal{F}_{N I D}\right\}$ attached to a robot arm (2). A five degrees of freedom electromagnetic tracker (3) with the reference frame $\left\{\mathcal{F}_{E M}\right\}$ is used to track the needle tip. The axial rotation of the needle is tracked using the motor encoder. A 3D ultrasound probe (4) with reference frame $\left\{\mathcal{F}_{U S}\right\}$ is used to track an artificial target in the phantom (5). Motion is applied to the phantom using a second robot arm (6) and motion compensation is performed using a force sensor (7) with the reference frame $\left\{\mathcal{F}_{F S}\right\}$. Frame $\left\{\mathcal{F}_{0}\right\}$ is the global reference frame.

Abolmaesumi et al. developed a tracking algorithm based on Star algorithm [20] and Kalman filter [21]. They used real-time 2D US images to track the carotid artery cross section. Guerrero et al. used an extended Kalman filter and an elliptical fitting model to determine the vessel boundary in real-time 2D US images [22]. The algorithm is tested using patient US images and provides a success rate of about 98 percent. Harris et al. tracked the 3D motion of liver using 3D cross-correlation-based speckle tracking algorithms in 4D US images [23]. Makhinya et al. developed a robust real-time algorithm based on optical flow to track the vessels in the liver in 2D US images [24]. Royer et al. developed a real-time tracking algorithm for deformable structures using 3D US images [25]. It was demonstrated that the algorithm can estimate the motion correctly in presence of different US shortcomings including speckle noise, large shadows and US gain variation, thanks to the consideration of US confidence map in the tracking process.

\section{B. Contributions}

In this letter, we present a robotic system along with a novel hybrid control framework which enables needle steering in a moving tissue while minimizing tissue damage. We propose a method to use force information coming from a force sensor at the base of the robotic system, which makes it feasible for clinical implementation. This hybrid framework uses the generic task functions to fuse targeting and motion compensation into a single control law. In order to evaluate the proposed method, insertions towards spherical targets, embedded in a gelatin phantom and biological tissue (bovine liver) are performed. We use US feedback and a Star algorithm to track the target and an electromagnetic tracker to accurately locate the tip of the needle. 


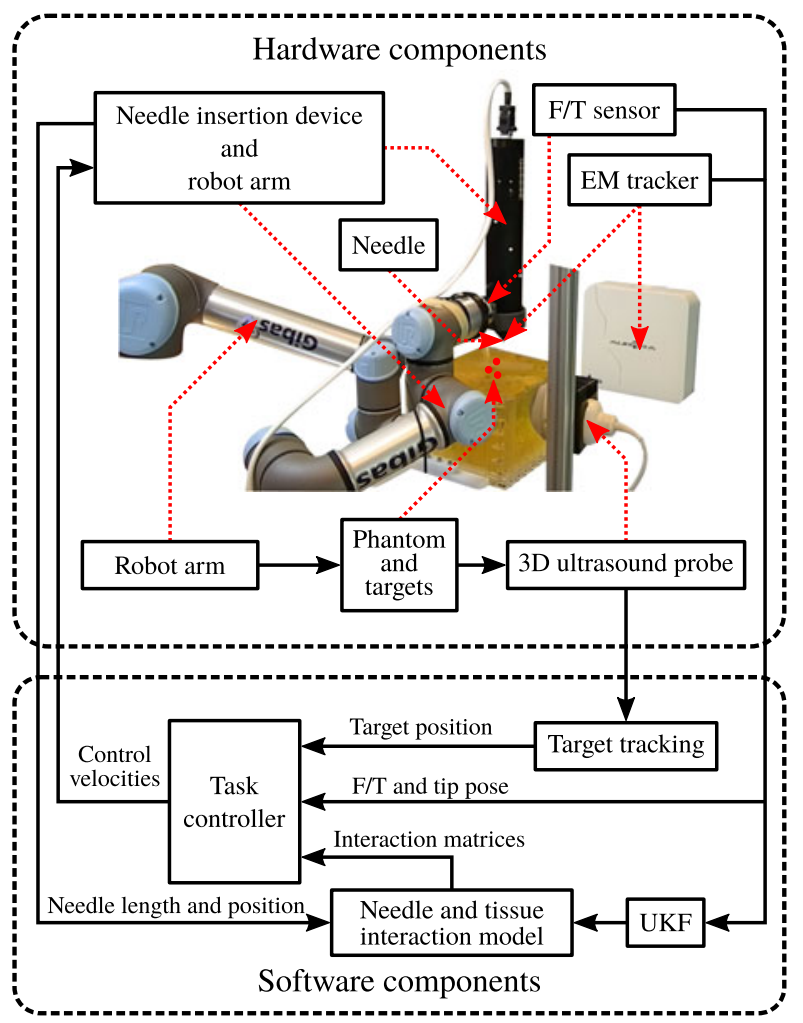

Fig. 2. Block diagram representing the elements of the setup and the global framework used for the insertion. Position of the target is tracked in ultrasound images. Measures from the force/torque $(\mathrm{F} / \mathrm{T})$ sensor and electromagnetic (EM) tracker are used as input to an unscented Kalman filter (UKF) to update the needle-tissue interaction model. The model and measures are used by a task controller to steer the needle tip towards the target while compensating for tissue motion.

\section{METHODS}

This section presents the tracking algorithm, control method and state estimation that we use to control a 6 DOF robotic manipulator holding a 2 DOF needle insertion device [26], as can be seen in Fig. 1. The overall framework for the control of the system is presented in Fig. 2.

\section{A. Ultrasound-Based Target Tracking}

A circular target, simulating a small cyst or a tumor, is tracked in 2D US images during the insertion process. We use a 3D US probe to acquire 3D US volumes. One 2D image is extracted from each of the volumes such that it is parallel to the $y-z$ plane of the probe frame $\left\{\mathcal{F}_{U S}\right\}$ (see Fig. 1). The tracking of the target center is done using a custom tracking algorithm described in Alg. 1 and illustrated in Fig. 3. Note that we considered spherical targets for validation, however the algorithm could be easily adapted to other target shapes. Knowing the pose of the US probe in the robot base frame $\left\{\mathcal{F}_{0}\right\}$ and the distance from the probe to the image plane, the position of the target is transformed from the image space to the robot base frame. This is then used as the reference target position for the controller loop described in next section.

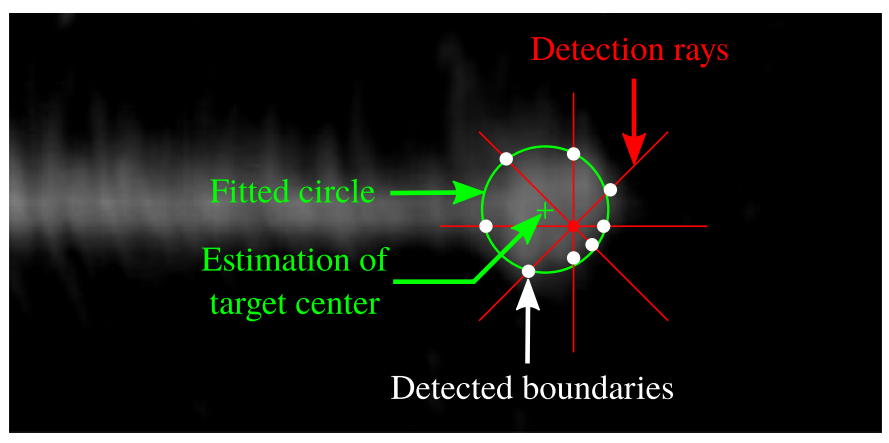

Fig. 3. Circular target detection algorithm using Star algorithm. The red dot is an initial guess of the target center from which rays are projected. The target center estimation (green cross) is obtained using circle fitting (green circle) on the detected boundaries along each ray (white dots).

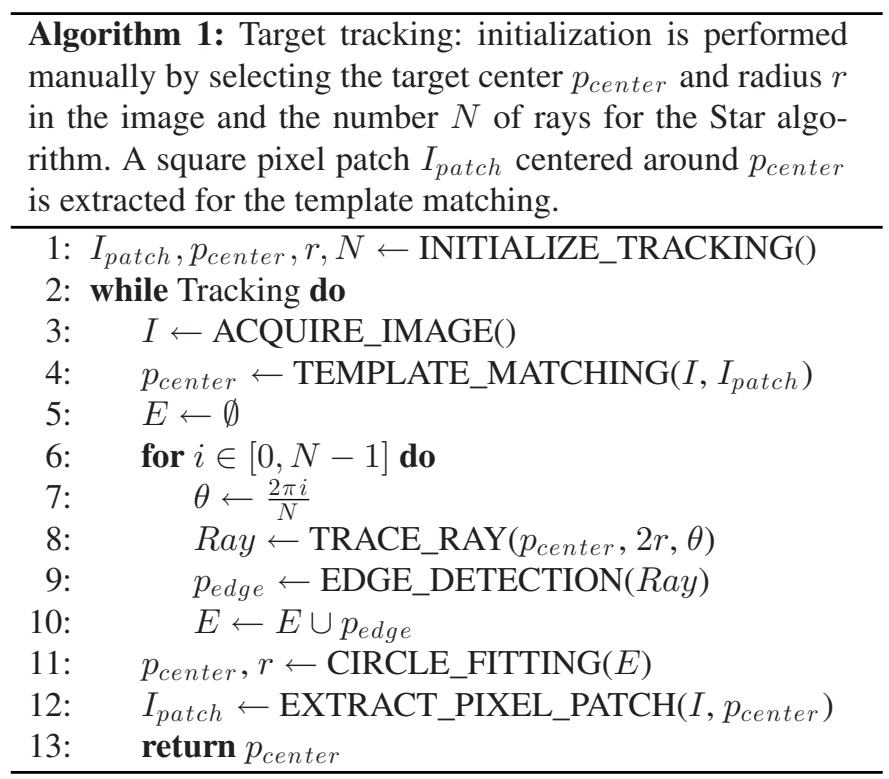

\section{B. Hybrid Control Framework}

The control of the 8 available DOF of the system is performed using a modified version of the method presented in [8]. We consider the following control vector $\left(\boldsymbol{v} \in \mathbb{R}^{8}\right)$ :

$$
\boldsymbol{v}=\left[\begin{array}{c}
\mathbf{v}_{b} \\
\boldsymbol{\omega}_{b} \\
v_{N I D} \\
w_{N I D}
\end{array}\right],
$$

where $v_{N I D}$ and $w_{N I D}$ denote the insertion and rotation velocities of the needle insertion device, respectively, and $\mathbf{v}_{b} \in \mathbb{R}^{3}$ and $\boldsymbol{\omega}_{b} \in \mathbb{R}^{3}$ correspond to the translational and angular velocity vectors of the tip of the insertion device, respectively. The last two are directly related to the velocities of the end-effector of the robot arm.

A task vector $\left(\boldsymbol{e}=\left[e_{1} \ldots e_{M}\right]^{T} \in \mathbb{R}^{M}\right)$ is defined such that it contains $M$ scalar tasks $\left(e_{i}\right)$ to be fulfilled. The desired value for the derivative of the task vector is defined as $\dot{e}_{d}=\left[\dot{e}_{1, d} \ldots \dot{e}_{M, d}\right]^{T}$, where each $\dot{e}_{i, d}$ is the desired value for 

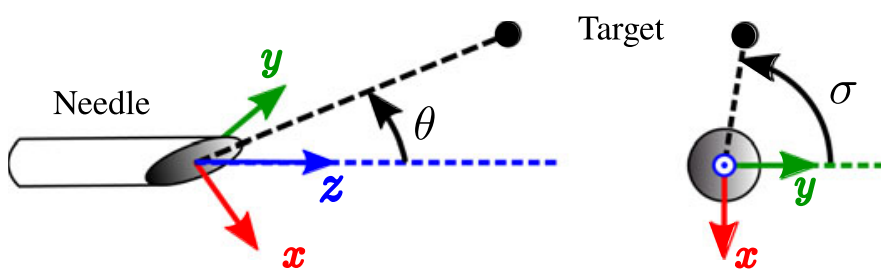

Fig. 4. Schematic of the sub-tasks $\left(e_{2}\right.$ and $\left.e_{3}\right)$ used for the steering of the needle. First one $\left(e_{2}\right)$ corresponds to the angle $(\theta)$ between the target and the needle axis, used to control the needle alignment at the beginning of the insertion. Second one $\left(e_{3}\right)$ corresponds to the angle $(\sigma)$ between the target and the bevel, used to orient the cutting edge of the bevel towards the target. The controller is designed such that both of them remains as close to zero as possible, ensuring accurate steering of the needle towards the target (see (6)-(8) and (9)-(11)).

$\dot{e}_{i}$. The control law is computed according to:

$$
\boldsymbol{v}=\boldsymbol{J}^{+} \dot{\boldsymbol{e}}_{d}
$$

where $^{+}$stands for the Moore-Penrose pseudo-inverse operator and $\boldsymbol{J} \in \mathbb{R}^{M \times 8}$ is the interaction matrix associated to the tasks, defined as the Jacobian matrix of the task vector with respect to the system inputs $(\boldsymbol{v})$ such that:

$$
\dot{e}=\frac{\partial e}{\partial t}=\boldsymbol{J} v
$$

1) Targeting Tasks Design: A targeting task made up of 3 scalar sub-tasks is defined to control the trajectory of the needle tip towards the previously defined target. The first sub-task $\left(e_{1}\right)$ is defined to control the velocity $\left(v_{t}\right)$ of the needle tip along the needle axis. The desired value for the sub-task variation is set to a predefined scalar constant insertion velocity $\left(\mathrm{v}_{t i p}\right)$ as long as the target is in front of the needle and the insertion is stopped when the target has been reached:

$$
\begin{gathered}
\dot{e}_{1}=v_{t}=\boldsymbol{J}_{v_{t}} \boldsymbol{v}, \\
\dot{e}_{1, d}=v_{t, d}=\left\{\begin{array}{cc}
\mathrm{v}_{t i p} & \text { if } z_{t}>0 \\
0 & \text { if } z_{t} \leq 0
\end{array},\right.
\end{gathered}
$$

where $z_{t}$ is the axial distance from the tip to the target and $\boldsymbol{J}_{v_{t}} \in \mathbb{R}^{1 \times 8}$ is the interaction matrix associated to the velocity of the needle tip along the needle axis $\left(v_{t}\right)$.

The second scalar sub-task $\left(e_{2}\right)$ is defined to control the angle $(\theta)$ between the needle tip axis and the target (see Fig. 4) such that:

$$
\begin{aligned}
e_{2} & =\theta=\operatorname{atan} 2\left(z_{t}, \sqrt{x_{t}^{2}+y_{t}^{2}}\right), \\
\dot{e}_{2} & =\dot{\theta}=\boldsymbol{J}_{\theta} \boldsymbol{v}, \\
\dot{e}_{2, d} & =\dot{\theta}_{d}=-\lambda_{\theta} \theta,
\end{aligned}
$$

where $x_{t}, y_{t}$ and $z_{t}$ are the coordinates of the position of the target expressed in the frame of the needle tip, $\boldsymbol{J}_{\theta} \in \mathbb{R}^{1 \times 8}$ is the interaction matrix associated to the angle $(\theta)$ and $\lambda_{\theta}$ is a positive control gain that tunes the exponential decrease rate of $\theta$.

The third scalar sub-task $\left(e_{3}\right)$ is used to control the orientation of the bevel such that it points towards the target. It is defined such that the angle $(\sigma)$ between the target and the cutting edge of the bevel is regulated to zero (see Fig. 4):

$$
\begin{aligned}
e_{3} & =\sigma=\operatorname{atan} 2\left(y_{t}, x_{t}\right)-\frac{\pi}{2}, \\
\dot{e}_{3} & =\dot{\sigma}=\boldsymbol{J}_{\sigma} \boldsymbol{v}, \\
\dot{e}_{3, d} & =\dot{\sigma}_{d}=-\lambda_{\sigma} \sigma,
\end{aligned}
$$

where $\boldsymbol{J}_{\sigma} \in \mathbb{R}^{1 \times 8}$ is the interaction matrix associated to the angle $(\sigma)$ and $\lambda_{\sigma}$ is a positive control gain that tunes the exponential decrease rate of $\sigma$.

It can be observed that both control inputs $\left(\omega_{b, z}\right.$ and $\left.\omega_{N I D}\right)$ defined in (1) result in the same rotation of the needle around its axis. Using $\omega_{b, z}$ leads to a rotation of the whole NID with the needle fixed inside, while using $\omega_{N I D}$ leads to a rotation of the needle inside the NID with the NID staying immobile. The former leads to unnecessary robot motion and increases the risk of collision with the environment or unfeasible robot configurations. Therefore, a scalar task $\left(e_{4}\right)$ is added to remove $\omega_{b, z}$ from the control, such that:

$$
\begin{aligned}
\dot{e}_{4} & =\omega_{b, z}=\left[\begin{array}{llllllll}
0 & 0 & 0 & 0 & 0 & 1 & 0 & 0
\end{array}\right] \boldsymbol{v}, \\
\dot{e}_{4, d} & =0 .
\end{aligned}
$$

It can be noted that the first and third targeting sub-tasks $\left(e_{1}\right.$ and $e_{3}$ ) only control the DOF of the tip that are used for pure tip-based steering, namely the insertion velocity and rotation velocity around the needle shaft. Sub-task $\left(e_{2}\right)$, on the contrary, can control the lateral translations of the tip and the orientation of the needle axis. Hence we further focus on this sub-task $\left(e_{2}\right)$ in the following.

2) Hybrid Control: In practice it can be observed that the interaction matrices $\left(\boldsymbol{J}_{v_{t}}\right.$ and $\left.\boldsymbol{J}_{\sigma}\right)$, corresponding to the sub-tasks $\left(e_{1}\right.$ and $\left.e_{3}\right)$ and defined in (4) and (10), have little dependence on the insertion depth. On the contrary the interaction matrix $\left(\boldsymbol{J}_{\theta}\right)$ of the second sub-task $\left(e_{2}\right)$ defined in (7) highly depends on the insertion depth since lateral translations and rotations of the tip become harder to control as the needle progresses inside the tissue. This sub-task $\left(e_{2}\right)$ tends to rapidly induce large control velocities once the needle is inserted deep in the tissue. Hence, we remove $e_{2}$ from the task list after a certain insertion depth has been reached such that only the tasks corresponding to pure tip-based control remain for the targeting.

The four sub-tasks defined previously ((4)-(13)) are sufficient to reach a target in stationary tissue. However tissue can move during the insertion because of physiological motion of the patient. If the needle does not follow the motion of the tissue this can damage the tissue surface due to tearing forces. In order to address this issue, we need to add a motion compensation task to minimize the forces exerted on the tissue.

3) Motion Compensation: At equilibrium, the total force exerted by the needle on the tissue is equal to the force exerted by the insertion device on the needle. We define a task $\left(e_{5} \in \mathbb{R}^{2}\right)$ to reduce the lateral force $\left(\boldsymbol{f}_{\text {lat }} \in \mathbb{R}^{2}\right)$ applied to the needle base ( $x$ and $y$ axis of frame $\left\{\mathcal{F}_{N I D}\right\}$ depicted in Fig. 1):

$$
\begin{aligned}
\dot{e}_{5} & =\dot{\boldsymbol{f}}_{l a t}=\boldsymbol{J}_{f} \boldsymbol{v}, \\
\dot{e}_{5, d} & =\dot{\boldsymbol{f}}_{l a t, d}=-\lambda_{f} \boldsymbol{f}_{l a t},
\end{aligned}
$$


where $\boldsymbol{J}_{f} \in \mathbb{R}^{2 \times 8}$ is the interaction matrix corresponding to the lateral force $\left(\boldsymbol{f}_{\text {lat }}\right)$ and $\lambda_{f}$ is a positive control gain that tunes the exponential decrease rate of $\boldsymbol{f}_{\text {lat }}$. The global interaction matrix $(\boldsymbol{J})$ defined in (2) is built by stacking the interaction matrices associated to each sub-task. Therefore online estimates of the matrices $\left(\boldsymbol{J}_{v_{t}}, \boldsymbol{J}_{\theta}, \boldsymbol{J}_{\sigma}\right.$ and $\left.\boldsymbol{J}_{f}\right)$ are required to compute the final control law (2). These matrices are computed using a finite difference method with a mechanics-based model of the interaction between the needle and the tissue. The model and its associated online update method are described next.

\section{Needle Insertion Modeling}

We use a mechanics-based model of needle insertion in soft tissue and an algorithm based on unscented Kalman filter (UKF) to update online the model using the available measures [27]. The model mainly consists of two parts interacting with each other, one 3D curve defining the needle shape and one 3D curve defining the shape of the path that has been cut in the tissue by the needle tip. In the case of patient motion, the lateral position ( $\mathrm{x} \in \mathbb{R}^{2}$ ) of the $3 \mathrm{D}$ curve representing the tissue needs to be updated to take into account the displacement of the real tissue. We adapt the update method to the measurements $\left(y \in \mathbb{R}^{12}\right)$ available in our experiments:

$$
\mathbf{y}=\left[\begin{array}{c}
f \\
t \\
p_{t} \\
d_{t}
\end{array}\right]
$$

where $\boldsymbol{f} \in \mathbb{R}^{3}$ and $\boldsymbol{t} \in \mathbb{R}^{3}$ are the forces and torques exerted at the needle base, and $\boldsymbol{p}_{t} \in \mathbb{R}^{3}$ and $\boldsymbol{d}_{t} \in \mathbb{R}^{3}$ are the position and direction of the needle tip, respectively. Measures are obtained using a force/torque sensor and a 5 DOF electromagnetic tracker placed inside the tip of the needle as described in Section III-A. The evolution and measure equations of the system can be written as:

$$
\begin{aligned}
& \mathbf{x}(k+1)=\mathbf{x}(k)+\boldsymbol{w}(k), \\
& \mathbf{y}(k)=\boldsymbol{h}(\mathbf{x}(k), k)+\boldsymbol{n}(k),
\end{aligned}
$$

with $k$ the time index, $\boldsymbol{w} \in \mathbb{R}^{2}$ the process noise, $\boldsymbol{n} \in \mathbb{R}^{12}$ the measure noise and $\boldsymbol{h}$ the function relating the tissue motion to the measures. A random walk model is used in the state equation (17) to account for any kind of motion. One advantage of the UKF is that it does not require an analytic formulation of $\boldsymbol{h}$ to compute the Kalman gain, as long as there exist a numerical method to compute the measures from the states. In our case, we use our numerical model to obtain the estimate of the measures and compute the Kalman gain. The estimates of $\boldsymbol{p}_{t}$ and $\boldsymbol{d}_{t}$ are computed as the position and direction of the tip of the 3D needle curve, respectively. The estimates of $f$ and $t$ are computed from the curvature of the $3 \mathrm{D}$ needle curve at the base and the mechanical properties of the needle (Young's modulus and section geometry). Between two iterations of the UKF, the model of the needle is also updated using the pose of the NID and the length of the needle outside the NID. Therefore function $\boldsymbol{h}$ is different at each time step $(k)$.

\section{EXPERIMENTS}

In this section, we present experiments performed to evaluate the US tracking, the control algorithm, and the motion compensation algorithm. We first describe the components of the experimental setup used to steer the needle. Subsequently, the experimental plan and results are presented, and we finish this section with a discussion on the results.

\section{A. Experimental Setup}

The experimental setup used to evaluate the overall system is shown in Fig. 1. It consists of a needle insertion device (NID), two robotic manipulators, an US probe, an electromagnetic (EM) tracker, a force/torque (F/T) sensor and a phantom. The NID has 2 DOF, which controls the insertion and rotation of the needle [26]. A Raspberry Pi 2 B (Raspberry Pi foundation, Caldecote, United Kingdom) along with a Gertbot motor controller board (Fen logic limited, Cambridge, United Kingdom) is used to control the robot through pulse-width-modulation (PWM). The two serial manipulators are UR3 and UR5 (Universal Robots A/S, Odense, Denmark). UR3 is a compact table-top robot to which the NID is connected through a plastic link. UR3 controls the position and orientation of the NID in 3D space. UR5 is a larger version of UR3, and therefore, it is used to apply motion to the phantom. The F/T sensor used in the experiments is ATI Nano 43 (ATI Industrial Automation, Apex, USA), which measures the outputting forces and torques from all three Cartesian coordinates (six-axis sensor). The forces and torques are measured with a resolution of $1.95 \mathrm{mN}$ and $25 \mathrm{mN}$.mm, respectively. The sensor is mounted between the robotic arm and the NID in order to measure the interaction forces. A registration step is performed to estimate the mass and position of the center of mass of the NID, as well as the biases of the sensor. During the experiments the effect of gravity and the biases are subtracted from the measurements depending on the device pose. Geometric transformation is applied to the sensor measures (frame $\left\{\mathcal{F}_{F S}\right\}$ in Fig. 1) in order to get the equivalent forces and torques applied at the needle base (frame $\left\{\mathcal{F}_{N I D}\right\}$ ) as required in (15) and (16). UR3, UR5 and the F/T sensor are all controlled through Ethernet (TCP/IP protocol) using Robot Operating System (ROS) (Open Source Robotics Foundation, Mountain View, USA). The EM tracking system is Aurora v3 EM tracker (Northern Digital Inc., Waterloo, Canada). The EM tracker measures the 3D position, pitch and yaw angles with an root mean square (RMS) error of $0.7 \mathrm{~mm}$ and $0.20^{\circ}$, respectively. A commercially available needle with a diameter of $0.55 \mathrm{~mm}(23.5 \mathrm{G})$ is equipped with a 5 DOF EM sensor in order to track the needle tip. The needle is hollow and it is made of stainless steel DIN 1.4301/AISI 304. The EM sensor is integrated within the needle, close to the tip. A preliminary registration step is performed to find the position of the tracker in the robot frame $\left\{\mathcal{F}_{0}\right\}$ and the offset between the center of the sensor and the tip of the needle. A least squares minimization is used between two sets of poses of the needle tip. Each set is obtained using either the EM sensor or the robot odometry. The motor encoder is used to measure the rotation about needle axis. The needle tip pose is measured 20 times per second. The 
US system is a Siemens Acuson S2000 (Siemens AG, Erlangen, Germany). Siemens 7CF1 Convex Volume 4D/3D transducer is used, which works at a frequency range of 1.0-7.0 Mhz. The resolution and the refresh rate of the system depends on the field of view, depth and sweeping angle of the probe. The pose of the US probe is registered in the robot frame $\left\{\mathcal{F}_{0}\right\}$ using least squares point cloud matching between two sets of positions of the needle tip measured after short insertions at different locations on the surface of the phantom. Each set is obtained using either the robot odometry or manual segmentation of the needle tip in acquired US volumes. Two phantoms are used for the needle steering experiments. The first phantom is made by mixing $14.9 \%$ (by-weight) porcine gelatin powder (Dr. Oetker, Ede, The Netherlands) with $85.1 \%$ water. This mixture results in a phantom with a Young's modulus of $35 \mathrm{kPa}$. The second phantom is a piece of bovine liver embedded in gelatin. The targets are spheres of different sizes, ranging from 4 to 8 millimeters, made of Play-Doh, which is easily moldable and gives good contrast in US images.

\section{B. Experimental Plan}

In order to evaluated the proposed needle steering method combined with motion compensation, we use three experimental cases.

Case I: In the first experimental case, we evaluate the proposed US target tracking algorithm. A 2D translational motion is applied to the phantom using UR5. The motion mimics the displacement of the liver during breathing [23] with the following profile:

$$
\boldsymbol{m}(t)=\boldsymbol{a}+\boldsymbol{b} \cos ^{2}\left(\frac{\pi}{T} t-\frac{\pi}{2}\right),
$$

where $\boldsymbol{a} \in \mathbb{R}^{3}$ is the initial position of the target, $\boldsymbol{b} \in \mathbb{R}^{3}$ is the magnitude of the motion and $T$ is the period of the motion. The magnitude of the motion is $15 \mathrm{~mm}$ and $7 \mathrm{~mm}$ in $x$ and $z$ direction of the global reference frame $\left\{\mathcal{F}_{0}\right\}$ depicted in Fig. 1, respectively. The period of the motion $(T)$ is set to 5 seconds. The measured positions of five targets are compared with the ground-truth obtained from the odometry of UR5.

Case II: The second experimental case is used to compare the applied forces and torques at the needle base between three different needle steering methods, each used during four needle insertions. The three methods are as follows. (1) Basemanipulation with no constraint on lateral motion of the needle at the insertion point. The needle is fully outside the NID. (2) Base-manipulation with remote-center-of-motion at the insertion point, i.e. the NID axis is always aligned with the insertion point. This method tries to minimize the lateral motion while the needle is fully outside the NID. (3) The NID tip is placed at the insertion point. The robot can only rotate around the insertion point. The needle is not fully outside the NID and it is supported by the NID body outside the phantom.

Case III: In the third experimental case, the needle is automatically steered towards a spherical target during ten needle insertions. The target location is registered to the UR3 reference frame using a 3D scan of the phantom with the 3D US probe. The target is selected manually at the beginning of each
TABLE I

The Results of the ExPERIMENTAL CASE I ARE PRESENTED

\begin{tabular}{|c|c|c|c|c|c|}
\hline \multirow[t]{2}{*}{$\#$} & \multicolumn{3}{|c|}{ Target position (mm) } & \multirow{2}{*}{$\begin{array}{l}\text { Error } \\
(\mathrm{mm})\end{array}$} & \multirow{2}{*}{$\begin{array}{l}\text { Mean } \\
(\mathrm{mm})\end{array}$} \\
\hline & $\mathrm{x}$ & $\mathrm{y}$ & $\mathrm{z}$ & & \\
\hline 1 & -17.5 & 4.9 & 64.4 & $0.9 \pm 0.5$ & $0.7 \pm 0.7$ \\
\hline 2 & -18.3 & 4.9 & 44.0 & $0.7 \pm 0.6$ & \\
\hline 3 & -13.5 & 11.6 & 24.3 & $0.6 \pm 0.4$ & \\
\hline 4 & -18.4 & 7.5 & 54.2 & $0.7 \pm 0.6$ & \\
\hline 5 & -30.6 & 10.9 & 34.3 & $0.6 \pm 0.4$ & \\
\hline
\end{tabular}

The initial target location in the ultrasound probe frame $\left\{\mathcal{F}_{U S}\right\}$ (Fig. 1) is indicated for each experiment. The error is calculated as the mean over time of the absolute distance between the position of the target obtained by the tracking and by the robot odometry. The error is calculated after compensating for the delay introduced by the image acquisition.

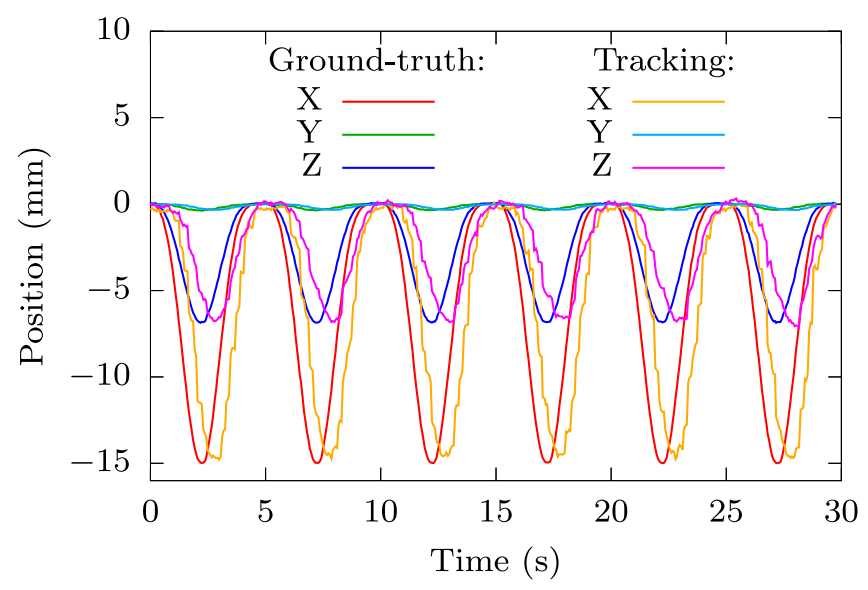

Fig. 5. Result of a representative experiment for the target tracking algorithm performance (case I). The motion described by (19) is applied to the gelatin phantom with a period $T=5 \mathrm{~s}$. Mean tracking error is $3.6 \mathrm{~mm}$ for this experiment and reduces to $0.6 \mathrm{~mm}$ after compensating for the delay of about $450 \mathrm{~ms}$ introduced by data acquisition.

TABLE II

The Results of the EXPerimental CASe II ARE Presented

\begin{tabular}{cccccc}
\hline \hline \multirow{2}{*}{ Method } & \multicolumn{4}{c}{ Force $(\mathrm{mN})$} & Mean force $(\mathrm{mN})$ \\
\cline { 2 - 4 } & $\# 1$ & $\# 2$ & $\# 3$ & $\# 4$ & \\
\hline (1) & 68 & 37 & 73 & 46 & $56 \pm 58$ \\
(2) & 28 & 68 & 59 & 56 & $53 \pm 49$ \\
(3) & 80 & 35 & 154 & 77 & $87 \pm 72$ \\
\hline \hline
\end{tabular}

Four insertions towards different target locations are performed for each method. The force is calculated for each experiment as the mean over time of the absolute lateral force. The mean force is the mean over the four experiments.

experiment, and the registration is done automatically. Similar to Case I, the UR5 moves the phantom with the same motion profile, but with different period $(T)$. The EM tracker, which is registered to the UR3 frame as well, is used to track the needle tip pose. The force/torque measurements are used to minimize the lateral forces at the insertion point. The steering method (3), explained in Case II, is used in this experimental case. In case a failure of the tracking algorithm is visible, the system can be stopped at any time by the operator and the needle is then automatically retracted from the tissue. 


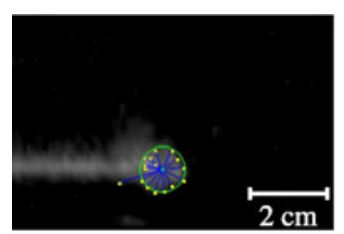

(a)

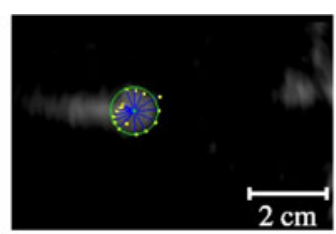

(b)

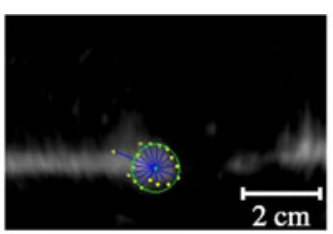

(c)

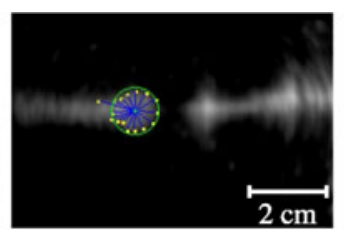

(d)

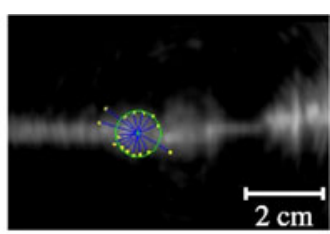

(e)

Fig. 6. Illustration of a sequence of ultrasound (US) images during a representative needle insertion in gelatin phantom. The US probe is fixed and a motion is applied to the phantom that simulates motion of a liver due to breathing. The position of the target is tracked in the images using a Star algorithm. The blue lines

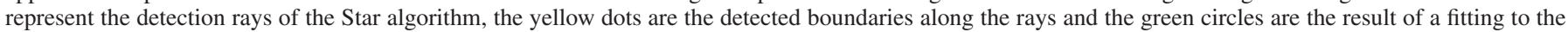
boundaries. The needle being inserted in the gelatin can be seen coming from the right. (a) $t=18.6 \mathrm{~s}(\mathrm{~b}) \mathrm{t}=24.9 \mathrm{~s}(\mathrm{c}) \mathrm{t}=30.2 \mathrm{~s}(\mathrm{~d}) \mathrm{t}=33.9 \mathrm{~s}$ (e) $\mathrm{t}=37.2 \mathrm{~s}$.

TABLE III

The Results of the EXPERIMENTAL CASE III ARE PRESENTED

\begin{tabular}{|c|c|c|c|c|c|c|c|}
\hline \multirow[b]{2}{*}{ Phantom type } & \multirow[b]{2}{*}{ \# } & \multirow[b]{2}{*}{ Period $(T(\mathrm{~s}))$} & \multicolumn{3}{|c|}{ Target position (mm) } & \multirow[b]{2}{*}{ Error $(\mathrm{mm})$} & \multirow[b]{2}{*}{ Mean error $(\mathrm{mm})$} \\
\hline & & & $\mathrm{x}$ & $\mathrm{y}$ & $\mathrm{z}$ & & \\
\hline \multirow[t]{5}{*}{ Gelatin } & 1 & 20 & 2.0 & 3.8 & 68.9 & 1.9 & \multirow[t]{5}{*}{$1.2 \pm 0.8$} \\
\hline & 2 & 20 & 1.8 & 0.8 & 57.8 & 0.7 & \\
\hline & 3 & 15 & 1.9 & -3.6 & 57.9 & 0.3 & \\
\hline & 4 & 10 & -2.3 & -3.9 & 57.1 & 2.2 & \\
\hline & 5 & 10 & -7.2 & 0.5 & 57.8 & 0.9 & \\
\hline \multirow[t]{5}{*}{ Liver } & 1 & 10 & -1.2 & -3.4 & 42.5 & 1.7 & \multirow[t]{5}{*}{$2.5 \pm 0.7$} \\
\hline & 2 & 10 & -0.8 & 3.5 & 42.2 & 2.3 & \\
\hline & 3 & 10 & 4.6 & -0.3 & 39.7 & 2.9 & \\
\hline & 4 & 10 & 5.1 & 6.0 & 40.1 & 2.0 & \\
\hline & 5 & 10 & 1.1 & 6.0 & 39.8 & 3.5 & \\
\hline
\end{tabular}

Five experiments are performed for each of two phantoms, one with gelatin and one with ex-vivo liver. The motion described by (19) is applied to the phantom with period $(T)$. The target location in initial tip frame is indicated for each experiment. The error is calculated as the absolute lateral distance between the needle tip axis and the center of the target at the end of the insertion. The mean and standard deviation of the error for each kind of phantom are presented separately.

\section{Results and Discussion}

In this section, we present the results of the three experimental cases. For Case I, five targets in different locations, depths and sizes are tracked with the proposed algorithm. Table I summarizes the target information, and the average error for each trial. The mean of tracking error is $0.7 \mathrm{~mm}$. The error is computed after compensating for the delay introduced by the image acquisition. Fig. 5 shows the ground-truth versus tracked location of the target for one of the trials. It is also visible from Fig. 5 that the process of acquiring a new US image, transferring it to the computer and applying the target tracking algorithm introduces a latency of about $450 \mathrm{~ms}$. A new volume is acquired every $110 \mathrm{~ms}$ and target tracking needs approximately $300 \mu \mathrm{s}$, which suggests that most of the latency is due to the conversion of the US volume from pre-scan to post-scan and transferring the images to the computer. The latency is reduced to $350 \mathrm{~ms}$ in Case III by reducing the field of view of the US transducer. A tracking sequence during a needle insertion corresponding to Case III is shown in Fig. 6.

The results of experimental Case II are presented in Table II. Four experiments are performed for each method, and the results are reported as the mean of absolute lateral forces calculated over time. The mean force values show that using method (3)induces greater lateral forces. This is due to the compliance of the needle, which affects methods (1) and (2). In method (3) the needle is supported with the NID and, therefore, it cannot bend outside the phantom. This results in the NID motion being applied directly onto the gelatin. On the other hand, in methods (1) and (2) the flexible needle outside the phantom is not supported and can easily bend. This compliant connection between the NID and the insertion point results in lower lateral forces.

In experimental Case III, the needle is steered towards ten spherical targets, five embedded in gelatin phantom and five embedded in bovine liver. The experiments results are presented in Table III. The targeting error is calculated as the absolute lateral distance between the needle and the center of the target. The mean targeting error is $1.2 \pm 0.8 \mathrm{~mm}$ and $2.5 \pm 0.7 \mathrm{~mm}$ for gelatin phantom and liver phantom, respectively. The mechanical properties of the gelatin phantom is known prior to the experiments and it is almost constant because of the homogeneity. The properties of liver is not precisely known and it also differs in different parts because of the heterogeneity. Furthermore, target tracking is more challenging in liver with respect to gelatin. These two issues directly influence the outcome of Case III experiments. Therefore, the needle steering is performed more accurately in gelatin rather than the liver.

\section{CONCLUSION AND Future WORK}

In this letter we have presented a control algorithm for a 2 DOF needle insertion device attached to a 6 DOF robot arm. Task functions are used to perform the steering of the needle tip towards a target at the same time as the compensation of 
lateral motions of the tissue. The controller uses the information provided on the needle by an EM tracker and a force sensor as well as the information provided on the target position by an US probe. The mean targeting accuracy obtained across several insertions in a moving bovine liver is $2.5 \pm 0.7 \mathrm{~mm}$, which is sufficient for most clinical needle insertion applications.

A delay compensation method could be used in the future to take into account the latency introduced by the acquisition of $3 \mathrm{D}$ US volumes and to further improve the steering accuracy. The registration of the US probe and EM tracker frames in the robot frame could also have an effect on the final targeting accuracy. Future work will thus include the usage of online 3D US volume feedback to directly measure the relative error between the needle tip and the target in the volume frame. This would eliminate the requirement for an accurate registration of the pose of the US probe. Furthermore, the developed control algorithm should be tested on live animals to validate its performance in a clinical context with real respiratory motions.

\section{REFERENCES}

[1] M. D. Cham, M. E. Lane, C. I. Henschke, and D. F. Yankelevitz, "Lung biopsy: Special techniques," in Seminars in Respiratory and Critical Care Medicine, vol. 29, no. 4. Thieme Medical Publishers, Aug. 2008, pp. 335349.

[2] S. DiMaio and S. Salcudean, "Needle steering and motion planning in soft tissues," IEEE Trans. Biomed. Eng., vol. 52, no. 6, pp. 965-974, Jun. 2005.

[3] D. Glozman and M. Shoham, "Image-guided robotic flexible needle steering," IEEE Trans. Robot., vol. 23, no. 3, pp. 459-467, Jun. 2007.

[4] M. Abayazid, G. Vrooijink, S. Patil, R. Alterovitz, and S. Misra, " Experimental evaluation of ultrasound-guided 3D needle steering in biological tissue," Int. J. Comput. Assisted Radiol. Surg., vol. 9, pp. 931-939, 2014. [Online]. Available: http://dx.doi.org/10.1007/s11548-014-0987-y

[5] D. Minhas, J. Engh, M. Fenske, and C. Riviere, "Modeling of needle steering via duty-cycled spinning," in Proc. 29th Annu. Int. Conf. IEEE Eng. Med. Biol. Soc., Aug. 2007, pp. 2756-2759.

[6] G. J. Vrooijink, M. Abayazid, S. Patil, R. Alterovitz, and S. Misra, "Needle path planning and steering in a three-dimensional non-static environment using two-dimensional ultrasound images," Int. J. Robot. Res., vol. 33, no. 10, pp. 1361-1374, 2014. [Online]. Available: http://dx.doi.org/10.1177/0278364914526627

[7] N. Shahriari, R. J. Roesthuis, N. J. van de Berg, J. J. van den Dobbelsteen, and S. Misra, "Steering an actuated-tip needle in biological tissue: Fusing FBG-sensor data and ultrasound images," in Proc. IEEE Int. Conf. Robot. Autom., May 2016, pp. 4443-4449.

[8] J. Chevrie, A. Krupa, and M. Babel, "Needle steering fusing direct base manipulation and tip-based control," in Proc. IEEE Int. Conf. Robot. Autom., May 2016, pp. 4450-4455.

[9] R. Ginhoux, J. Gangloff, M. de Mathelin, L. Soler, M. M. A. Sanchez, and J. Marescaux, "Active filtering of physiological motion in robotized surgery using predictive control," IEEE Trans. Robot., vol. 21, no. 1, pp. 67-79, Feb. 2005.

[10] S. G. Yuen, D. P. Perrin, N. V. Vasilyev, P. J. d. Nido, and R. D. Howe, "Force tracking with feed-forward motion estimation for beating heart surgery," IEEE Trans. Robot., vol. 26, no. 5, pp. 888-896, Oct. 2010.
[11] S. F. Atashzar, I. Khalaji, M. Shahbazi, A. Talasaz, R. V. Patel, and M. D. Naish, "Robot-assisted lung motion compensation during needle insertion," in Proc. IEEE Int. Conf. Robot. Autom., May 2013, pp. 1682 1687.

[12] Y. J. Kim, J. H. Seo, H. R. Kim, and K. G. Kim, "Impedance and admittance control for respiratory-motion compensation during robotic needle insertion-A preliminary test," Int. J. Med. Robot. Comput. Assisted Surg., vol. 13, no. 4, Dec. 2017. [Online]. Available: http://dx.doi.org/10.1002/rcs.1795

[13] Z. Neubach and M. Shoham, "Ultrasound-guided robot for flexible needle steering," IEEE Trans. Biomed. Eng., vol. 57, no. 4, pp. 799-805, Apr. 2010

[14] M. Abayazid, R. J. Roesthuis, R. Reilink, and S. Misra, "Integrating deflection models and image feedback for real-time flexible needle steering," IEEE Trans. Robot., vol. 29, no. 2, pp. 542-553, Apr. 2013.

[15] N. Shahriari, R. J. Roesthuis, N. J. van de Berg, J. J. van den Dobbelsteen, and S. Misra, "Steering an actuated-tip needle in biological tissue: Fusing FBG-sensor data and ultrasound images," in Proc. IEEE Int. Conf. Robot. Autom., 2016, pp. 4443-4449.

[16] H. R. S. Neshat and R. V. Patel, "Real-time parametric curved needle segmentation in 3D ultrasound images," in Proc. IEEE RAS EMBS Int. Conf. Biomed. Robot. Biomechatronics, Oct. 2008, pp. 670-675.

[17] P. Chatelain, A. Krupa, and M. Marchal, "Real-time needle detection and tracking using a visually servoed 3D ultrasound probe," in Proc. IEEE Int. Conf. Robot. Autom., May 2013, pp. 1676-1681.

[18] A. Pourtaherian, S. Zinger, P. H. N. de With, H. H. M. Korsten, and N. Mihajlovic, "Gabor-based needle detection and tracking in threedimensional ultrasound data volumes," in Proc. IEEE Int. Conf. Image Process., Oct. 2014, pp. 3602-3606.

[19] P. Chatelain, A. Krupa, and N. Navab, "3D ultrasound-guided robotic steering of a flexible needle via visual servoing," in Proc. IEEE Int. Conf. Robot. Autom., 2015, pp. 2250-2255.

[20] N. Friedland and D. Adam, "Automatic ventricular cavity boundary detection from sequential ultrasound images using simulated annealing," IEEE Trans. Med. Imag., vol. 8, no. 4, pp. 344-353, Dec. 1989.

[21] P. Abolmaesumi, S. E. Salcudean, W.-H. Zhu, M. R. Sirouspour, and S. P DiMaio, "Image-guided control of a robot for medical ultrasound," IEEE Trans. Robot. Autom., vol. 18, no. 1, pp. 11-23, Feb. 2002.

[22] J. Guerrero, S. E. Salcudean, J. A. McEwen, B. A. Masri, and S. Nicolaou, "Real-time vessel segmentation and tracking for ultrasound imaging applications," IEEE Trans. Med. Imag., vol. 26, no. 8, pp. 1079-1090, Aug. 2007

[23] E. J. Harris, N. R. Miller, J. C. Bamber, J. R. N. Symonds-Tayler, and P. M. Evans, "Speckle tracking in a phantom and feature-based tracking in liver in the presence of respiratory motion using $4 \mathrm{~d}$ ultrasound," Phys. Med. Biol., vol. 55, no. 12, pp. 3363-3380, 2010. [Online]. Available: http://stacks.iop.org/0031-9155/55/i=12/a=007

[24] M. Makhinya and O. Goksel, "Motion tracking in 2D ultrasound using vessel models and robust optic-flow," in Proc. MICCAI (Medical Image Computing and Computer Assisted Intervention) CLUST (Challenge on Liver Ultrasound Tracking), Munich, Germany, Oct. 2015, pp. 20-27.

[25] L. Royer, A. Krupa, G. Dardenne, A. Le Bras, É. Marchand, and M. Marchal, "Real-time target tracking of soft tissues in 3D ultrasound images based on robust visual information and mechanical simulation," Med. Image Anal., vol. 35, pp. 582-598, 2017.

[26] N. Shahriari, E. Hekman, M. Oudkerk, and S. Misra, "Design and evaluation of a computed tomography (CT)-compatible needle insertion device using an electromagnetic tracking system and CT images," Int. J. Comput. Assisted Radiol. Surg., vol. 10, no. 11, pp. 1845-1852, 2015.

[27] J. Chevrie, A. Krupa, and M. Babel, "Online prediction of needle shape deformation in moving soft tissues from visual feedback," in Proc. IEEE/RSJ Int. Conf. Intell. Robots Syst., Oct. 2016, pp. 2375-2380. 